(0.9-4.3) and 9.5 (5.3-13.3), $\mathrm{p}=0.000$. When patients tested positive in the ARIA, $\mathrm{ABT}$ and the 2-sided ELISA almost all patients had undetectable drug levels. Conclusions: The fast majority of the AS patients develop ADA. The ARIA detects more ADA compared to the assays susceptible for drug interference, the $\mathrm{ABT}$ and the more frequently used 2-sided ELISA. Immunogenicity data should be interpreted with the knowledge of the assay and in context of drug levels.

Disclosure of Interest: J. Ruwaard: None declared, A. Marsman: None declared, M. Nurmohamed: None declared, H. te Velthuis: None declared, K. Bloem: None declared, T. Rispens Speakers bureau: Pfizer, AbbVie, G. J. Wolbink Grant/research support from: Pfizer, Speakers bureau: Pfizer, AbbVie, UCB DOI: 10.1136/annrheumdis-2017-eular.6149

\title{
OP0026 HIGH DRUG-FREE REMISSION IN EARLY PERIPHERAL SPONDYLOARTHRITIS AFTER INDUCTION THERAPY WITH GOLIMUMAB
}

P. Carron ${ }^{1,2}$, G. Varkas ${ }^{1,2}$, H. Cypers ${ }^{1,2}$, L. Van Praet $^{1}$, D. Elewaut ${ }^{1,2}$, F. Van den Bosch ${ }^{1,2} .{ }^{1}$ Rheumatology, Univeristy of Ghent; ${ }^{2}$ VIB Inflammation Research Center, Ghent University, Gent, Belgium

Background: In rheumatoid arthritis there is accumulating evidence for a socalled "window of opportunity" remission induction treatment at an early stage of the disease. Whether a similar concept also applies to Spondyloarthritis is currently unknown. Given the higher chance of obtaining remission or low disease activity with early aggressive (biologic) treatment in early axial SpA, one could speculate that there is at least also a possibility of a "window of opportunity" for drug-free remission in peripheral SpA (pSpA).

Objectives: To evaluate sustained drug-free clinical remission after induction therapy with golimumab in patients with active peripheral Spondyloarthritis in a very early stage of the disease.

Methods: CRESPA (Clinical REmission in peripheral SPondyloArthritis) is an ongoing monocentric study of golimumab treatment in $\mathrm{pSpA}$ patients. Eligible patients were $\geq 18$ years and fulfilled the Assessment of SpondyloArthritis international Society (ASAS) classification criteria for pSpA. All patients had a symptom duration $<12$ weeks. Patients were randomized 2:1 to receive golimumab $50 \mathrm{mg}$ every 4 weeks or matching placebo for 24 weeks. The primary endpoint was the percentage of patients achieving clinical remission at week 24 , defined as the absence of arthritis, enthesitis and dactylitis on clinical examination. If patients were in clinical remission at two consecutive visits (major evaluations at week 12, 24, 36 and 48 ) treatment was stopped.

These patients were prospectively followed to assess the percentage of patients in sustained drug-free clinical remission, compared to those experiencing a clinical relapse of arthritis, dactylitis or enthesitis. In case of clinical relapse patients were retreated with open-label golimumab in the extension part of this trial.

Results: In total 60 patients were randomized of whom 20 received placebo and 40 golimumab. Baseline demographics and disease characteristics were generally similar between the 2 groups. At week 24 a significantly higher percentage of patients receiving golimumab achieved clinical remission compared to patients receiving placebo $(75 \%(30 / 40)$ versus $20 \%(4 / 20) ; \mathrm{P}<0.001)$. At week 12 similar results were observed $(70 \%(28 / 40)$ versus $15 \%(3 / 20)$; $P<0.001)$. In 49 out of the 60 patients treatment could be stopped because they fulfilled sustained clinical remission at 2 major consecutive visits. All patients had at least a follow up of 12 months after discontinuation of treatment with a maximum of follow-up of 58 months. $53 \%(26 / 49)$ of these patients are still in drug-free sustained clinical remission and $47 \%$ (23/49) had a clinical relapse after withdrawal. The majority of relapsed patients experienced their flare within 6 months after discontinuation. Conclusions: In patients with active, very early peripheral spondyloarthritis, treatment with golimumab led to high percentages of clinical remission at week 12 and 24. A remarkably high percentage of patients are still in sustained drug-free clinical remission after induction therapy with golimumab which indicates a window of opportunity for drug-free remission in this disease.

Disclosure of Interest: None declared

DOI: 10.1136/annrheumdis-2017-eular.1656 